\title{
Increased Epicardial Fat Thickness Is Associated with Cardiac Functional Changes in Healthy Women
}

\author{
Baris Kilicaslan', Oner Ozdogan', Mehmet Aydin'1, Huseyin Dursun', \\ Ibrahim Susam ${ }^{1}$ and Faruk Ertas ${ }^{2}$
}

${ }^{1}$ Department of Cardiology, Tepecik Research and Training Hospital, Izmir, Turkey

${ }^{2}$ Departmet of Cardiology, Dicle University Medical Faculty, Diyarbakir, Turkey

Epicardial fat tissue is a visceral fat depot with anatomical and functional contiguity to the myocardium and coronary arteries. The objective of this study was to evaluate the association between epicardial fat thickness (EFT) and cardiac changes in healthy female subjects. The study population consisted of ninety-six consecutive healthy female (mean age $31.1 \pm 6.7$ years) who underwent transthoracic echocardiography. EFT was measured by echocardiography. Subjects were divided into two groups according to the EFT; EFT $<6 \mathrm{~mm}$ and EFT $\geq 6 \mathrm{~mm}$. The cardiac structural changes, increased left atrial volume indices (LAVI) $(41.2 \pm 9.9$ vs. $52.6 \pm 12.5, p=0.001)$ and left ventricular mass indices (LVMI) (129.6 \pm 32.1 vs. $155.6 \pm 31.6 p<0.05)$, were observed in patients with increased EFT. Myocardial tei index (MTI), which was used to evaluate both systolic and diastolic functions, was higher with increased EFT (0.44 \pm 0.07 vs. $0.48 \pm 0.08, p=0.02$ ). The correlation analysis revealed significant correlation between EFT and each of LAVI $(r=0.312, p=0.002)$, LVMI $(r=0.301, p=0.003)$, body mass index (BMI) $(r=0.8, p<0.001)$, and MTI $(r=0.27, p=0.005)$. Multivariate regression analysis demonstrated that EFT was associated with $\operatorname{BMI}(t=5.28, p=0.001)$, MTI $(r=2.39, p=0.019)$, LVMI $(r=2.16, p=0.01)$, and LAVI $(r=3.21, p=0.002)$. In conclusion, EFT is an important predictor of cardiac alterations in women who are prone to obesity.

Keywords: epicardial fat tissue; left atrial volume index; left ventricular mass index; metabolic syndrome; myocardial Tei index

Tohoku J. Exp. Med., 2012 Oct, 228 (2), 119-124. C 2012 Tohoku University Medical Press

Epicardial fat tissue is a visceral fat depot with anatomical and functional contiguity to the myocardium and coronary arteries. Epicardial fat tissue's embryologic origin is similar to intra-abdominal visceral adipose tissue and owes to the splanchnopleuric mesoderm. Epicardial fat tissue also acts as an endocrine organ. Epicardial fat tissue has biochemical, mechanical, and biomolecular properties (Taguchi et al. 2001; Iacobellis et al. 2005a). Under pathological circumstances, epicardial fat can locally affect the heart and coronary arteries through vasocrine or paracrine secretion of proinflammatory cytokines (Iacobellis et al. 2003b; Ding et al. 2009). Many imaging techniques such as multi slice magnetic resonance imaging (MRI), MR spectroscopy, and computed tomography (CT) can demonstrate epicardial fat tissue. However, these imaging techniques are expensive and not routinely available and they may have contraindications in selected patient populations. Transthoracic echocardiography (TTE) has been validated as an easy and reliable method to quantify the presence of visceral adipose tissue by measuring the Epicardial fat thickness (EFT) (Iacobellis et al. 2003a, 2003b). Increased body mass index (BMI) and waist-hip ratio are also useful markers of cardiovascular risk (National Cholesterol Education Program 2002). EFT correlates well with metabolic syndrome (MS) and coronary artery disease incidence (Ahn et al. 2008). However, in healthy subjects, the relationship of EFT with cardiac systolic and diastolic functions and with cardiovascular risks is not well understood, and it needs more study before EFT is used as a tool for routine clinical assessment.

In this study, we sought to examine the relationship between EFT and cardiac structural and functional changes as observed by echocardiography. We also investigated the association of EFT with conventional cardiovascular risk factors in a healthy female population.

\section{Methods}

Study Population

Ninety-six consecutive healthy female (mean age $31.1 \pm 6.7$ years) who were admitted to our cardiology outpatient clinic were prospectively recruited in the study. The medical records of all patients were assessed. Subjects with history of established heart dis-

Received June 4, 2012; accepted August 20, 2012. Published online September 6, 2012; doi: 10.1620/tjem.228.119.

Correspondence: Baris Kilicaslan, M.D., Department of Cardiology, Tepecik Research and Training Hospital, Manavkuyu mahallesi 249/4 sok. no: 2 A blok D: 16 Bayrakl1, Izmir 35030, Turkey.

e-mail: kilicaslanbaris@yahoo.com 
ease, type II diabetes mellitus, hypertension, dyslipidemias, thyroid diseases, and active inflammation were excluded from the study. We tried to exclude the acute systemic inflammatory effect to avoid the confounding role of epicardial fat tissue. All subjects went to biochemical laboratory for blood test and transthoracic echocardiography for standard echocardiographic examination and to determine EFT. Our study subjects were divided into two groups regarding their EFTs. EFT $<6 \mathrm{~mm}$ and EFT $\geq 6 \mathrm{~mm}$.

The study protocol was approved by Tepecik Research and Training Hospital Ethics Committee and informed consent was obtained from all participants. No extramural funding was used to support this work. Investigation was conducted in accordance with the guidelines proposed in the Declaration of Helsinki.

\section{Anthromorphometry, blood pressure measurement and laboratory} tests

Body mass index (BMI) was calculated by dividing weight (in kilogram) divided by the square of height (in meter). Waist circumference (in centimeter) was measured from the midpoint between the lowest rib and the iliac crest while the subject was standing. After a 30-minute resting period, blood pressure (BP) was measured twice in both arms while sitting using a mercury sphygmomanometer and appropriately sized cuffs. The average of two higher BP measurements was used to calculate systolic and diastolic BP.

Blood samples were obtained after at least 12 hours of fasting. Levels of total cholesterol (Tchol), triglycerides (TG), low-density lipoprotein cholesterol (LDL), high-density lipoprotein cholesterol (HDL), glucose, hemoglobin, and creatinine were assayed by the routine laboratory techniques. Serum CRP levels were evaluated by nephelometry.

\section{Echocardiography}

Echocardiographic examinations were performed according to American Society of Echocardiography recommendations (Lang et al.
2005) with a Vivid 3 instrument (General Electric, Horten, Norway) and a $2.5 \mathrm{MHz}$ transducer. All echo-Doppler studies were carried out by the same observer, who was unaware of the clinical data to avoid inter-reader variability. Measurements were made on 3 representative beats and the average of the results was taken. Echocardiograms were recorded digitally to an online system for measurement and analysis. Standard echocardiographic analysis included two-dimensional M-mode, and Doppler flow measurements.

EFT was measured according to the method previously described (Iacobellis et al. 2003b). Epicardial fat tissue was defined as the echo-free space between the outer layer of the myocardial wall and the visceral layer of the pericardium (Iacobellis et al. 2003a, 2003b). We measured EFT on the free wall of right ventricle from the parasternal long-axis views at mid ventricle during end diastole (marked by the R wave on the ECG recording) for the mid-ventricular parasternal short axis assessment, maximum EFT was measured on the free wall of right ventricle along the mid-line of the ultrasound beam perpendicular to the interventricular septum to the mid-chordal and the tip of the papillary muscle level as the anatomic landmark (Fig. 1). In order to standardize the measuring point, we used the aortic annulus as the anatomical reference. The maximum values at each site were measured, and the average value was taken into consideration.

Left ventricular (LV) ejection fraction was measured with modified Simpson's rule from 2-dimension echocardiographic tracings obtained in apical 4-chamber view and other echocardiographic measurements were assessed according to American Society of Echocardiography guidelines. LV mass was calculated using the equation described by Devereux (Devereux and Reichek 1977). The LV mass index (LVMI) was calculated by dividing the LV mass by body surface area. Body surface area was calculated according to formula: [(weight $t^{0.425} \times$ height $\left.\left.^{0.725}\right) / 139.2\right]$. The left atrial volume was calculated using the area-length method. Using this method, the area of the left atrium was measured by planimetry of both apical views

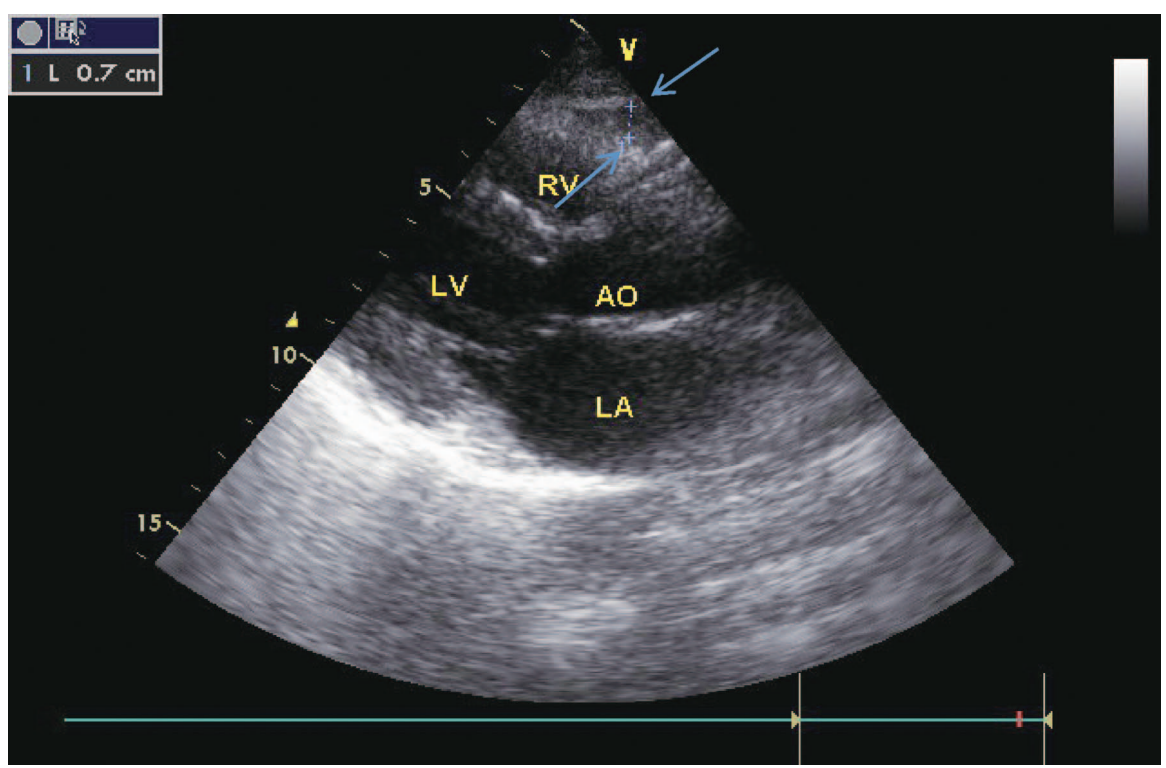

Fig. 1. An example of measurement of epicardial fat thickness. Epicardial fat was identified as an echo-free space in the pericardial layers on the 2-dimensional echocardiography, and its thickness was measured perpendicularly on the free wall of the right ventricle at end-diastole. Blue arrows indicate boundaries of epicardial fat tissue.

LA, Left atrium; LV, Left ventricle; RV, Right ventricle; AO, Aorta. 
(A1 and A2). A linear dimension was measured from the centre of the mitral annulus to the superior border of the chamber $(\mathrm{L})$. The left atrial volume was then calculated as $[(0.85 \times \mathrm{A} 1 \times \mathrm{A} 2) \div \mathrm{L}]$ (Pritchett et al. 2003). The left atrial volume index (LAVI) was calculated by dividing the left atrial volume by body surface area. Diastolic functions were evaluated by mitral inflow parameters (E, A, deceleration time [DT], E/A ratio) (Gottdiener et al. 2004).

Tissue Doppler echocardiography, guided by the two-dimensional 4-chamber view, a 5-mm sample volume was placed just apical to the medial and lateral mitral annulus and to the lateral tricuspid annulus, using pulsed-wave tissue TDI. The tissue Doppler-derived early diastolic annular velocity (e') and systolic annular velocity (sm) were measured from the average of septal and lateral tissue velocities in the apical 4-chamber view. Settings were adjusted for a frame rate between 120 and $180 \mathrm{~Hz}$, and a cineloop of 3 to 5 consecutive heart beats was recorded. TDI-derived systolic indices were measured from the mitral annulus. Myocardial Tei index (MTI) was calculated as the sum of isovolumic contraction time and isovolumic relaxation time divided by ejection time (Tei et al. 1995).

\section{Statistical Analysis}

Statistical analysis was performed using SPSS 11.0 for Windows. All data were expressed as mean \pm standard deviation and the median values for continuous variables and as percentage for categorical variables. $p<0.05$ indicates statistical significance. Patients' characteristics according to EFT were compared using an independent $t$-test and chi-square test. The EFT was also compared with other factors using independent samples $t$-test. The relationship between EFT and other variables was assessed using Pearson correlation analyses. Multivariate analysis was performed to determine the factors related to EFT.

\section{Results}

In the present series, the population's mean age was $31.1 \pm 6.7$ years. The mean value of the EFT was $6.03 \mathrm{~mm}$ (range 1.8 to $11.5 \mathrm{~mm}$ ). According to the mean EFT, we divided the study population into 2 groups (EFT $<6 \mathrm{~mm}$; EFT $\geq 6 \mathrm{~mm}$ ). There were no significant differences in the baseline characteristics, except for waist circumference $(p<0.05)$ and BMI $(p<0.05)$. The comparisons of anthropometric and echocardiographic parameters were shown in Table 1. There were significant differences in fasting glucose $(p=0.01)$, TG $(p=0.04), \mathrm{CRP}(p=0.005)$ and HDL levels $(p=0.01)$. The echocardiographic parameters of MTI $(p=0.02)$, LAVI $(p=0.001)$, and LVMI $(p<0.05)$ were higher in patients with increased EFT. The correlation of EFT with anthropometric and echocardiographic parameters was assessed by Pearson correlation test. A significant correlation was revealed between EFT and LAVI $(r=0.312$, $p=0.002)$, LVMI $(r=0.301, p=0.003)$, HDL $(r=-0.27$, $p=0.01), \mathrm{TG}(r=0.22, p=0.02), \mathrm{CRP}(r=0.39, p<0.001)$,

Table 1. Anthropometric and echocardiographic variables of the subjects.

\begin{tabular}{lccc}
\hline & EAT<6 $(n: 46)$ & EAT $\geq 6(n: 52)$ & $P$ \\
\hline Age (years) & $29.9 \pm 6.2$ & $32.1 \pm 6.9$ & 0.09 \\
Waist circumference (cm) & $89.5 \pm 9.6$ & $106.6 \pm 12.1$ & $\mathbf{0 . 0 0 1}$ \\
BMI (kg/m ${ }^{2}$ ) & $24.6 \pm 3.9$ & $33.6 \pm 6.1$ & $\mathbf{0 . 0 0 1}$ \\
Systolic TA (mmHg) & $113.8 \pm 13.4$ & $115.2 \pm 12.4$ & 0.59 \\
DiastolicTA (mmHg) & $75.5 \pm 8.4$ & $75.6 \pm 8.2$ & 0.91 \\
LVEF (\%) & $61.0 \pm 2.3$ & $61.3 \pm 2$ & 0.55 \\
LV mass indices & $129.6 \pm 32.1$ & $155.6 \pm 31.6$ & $\mathbf{0 . 0 5}$ \\
LA volume indices & $41.2 \pm 9.9$ & $52.6 \pm 12.5$ & $\mathbf{0 . 0 0 1}$ \\
Mitral DecT & $183.7 \pm 29.7$ & $192.0 \pm 36.3$ & 0.21 \\
Mitral Tei İndices & $0.44 \pm 0.07$ & $0.48 \pm 0.08$ & $\mathbf{0 . 0 2}$ \\
Mitral e' & $0.12 \pm 0.02$ & $0.11 \pm 0.03$ & 0.33 \\
Mitral Sm' & $0.08 \pm 0.01$ & $0.09 \pm 0.01$ & 0.37 \\
Tricuspid Tei indices & $0.23 \pm 0.07$ & $0.26 \pm 0.07$ & 0.14 \\
Tricuspid e' & $0.14 \pm 0.03$ & $0.13 \pm 0.03$ & 0.66 \\
Tricuspid Sm' & $0.13 \pm 0.03$ & $0.15 \pm 0.1$ & 0.08 \\
hs-CRP & $0.38 \pm 0.42$ & $0.62 \pm 0.41$ & $\mathbf{0 . 0 0 5}$ \\
Fasting Glucose (mg/dl) & $86.6 \pm 7.5$ & $90.8 \pm 9.4$ & $\mathbf{0 . 0 1}$ \\
TChol (mg/dl) & $189 \pm 35.8$ & $185.1 \pm 34$ & 0.58 \\
Triglyceride (mg/dl) & $97.6 \pm 40.4$ & $119.2 \pm 63.8$ & $\mathbf{0 . 0 4}$ \\
HDL (mg/dl) & $56.5 \pm 11.9$ & $50.3 \pm 9.8$ & $\mathbf{0 . 0 1}$ \\
LDL (mg/dl) & $116.2 \pm 24.7$ & $117.5 \pm 26.7$ & 0.81 \\
\hline
\end{tabular}

e', Tissue doppler-derived early diastolic annular velocity; Sm, Tissue doppler-derived systolic annular velocity; BMI, Body mass index; TA, Tension arterial; LVEF, Left ventricular ejection fraction; Dec T, Deceleration time; hs-CRP, High sensitive C-reactive protein; Tchol, Total cholesterol; HDL, High density lipoprotein cholesterol; LDL, Low density lipoprotein cholesterol 
Table 2. Correlations and multivariate regression analyses of anthropometric and laboratory variables with epicardial adipose tissue.

\begin{tabular}{lcl}
\hline & $R$ & $p$ \\
\hline Age (years) & 0.19 & 0.05 \\
Waist circumference (cm) & 0.75 & 0.001 \\
BMI (kg/m $\left.{ }^{2}\right)$ & 0.8 & 0.001 \\
Systolic TA (mmHg) & -0.06 & 0.55 \\
DiastolicTA (mmHg) & -0.09 & 0.35 \\
Fasting Glucose (mg/dl) & 0.26 & 0.008 \\
TChol (mg/dl) & -0.03 & 0.75 \\
Triglyceride (mg/dl) & 0.22 & 0.02 \\
hs-CRP & 0.38 & 0.001 \\
HDL (mg/dl) & -0.27 & 0.01 \\
\hline
\end{tabular}

Multivariate regresion analyses

\begin{tabular}{lcl}
\hline \multicolumn{1}{c}{$r^{2}=0.54$} & $T$ & $P$ \\
\hline Triglyceride $(\mathrm{mg} / \mathrm{dl})$ & 2.07 & 0.04 \\
hs-CRP & 0.75 & 0.45 \\
HDL $(\mathrm{mg} / \mathrm{dl})$ & 0.4 & 0.68 \\
BMI $(\mathrm{kg} / \mathrm{m} 2)$ & 5.28 & 0.001 \\
Fasting Glucose $(\mathrm{mg} / \mathrm{dl})$ & 0.96 & 0.34 \\
Waist circumference $(\mathrm{cm})$ & 10.07 & 0.001 \\
\hline
\end{tabular}

BMI, Body mass index; TA, Tension arterial; hs-CRP, High sensitive C-reactive protein; Tchol, Total cholesterol; HDL, High density lipoprotein; LDL, Low density lipoprotein.

Table 3. Correlations and multivariate regression analyses of the echocardiographic parameters with epicardial adipose tissue.

\begin{tabular}{lcl}
\hline & $r$ & $P$ \\
\hline LVEF (\%) & 0.03 & 0.74 \\
LA volume Index & 0.31 & 0.002 \\
Mitral DecT & 0.19 & 0.06 \\
Mitral Tei Index & 0.27 & 0.005 \\
Mitral e' & -0.19 & 0.04 \\
Mitral Sm' & 0.08 & 0.4 \\
Tricuspid Tei Index & 9.18 & 0.06 \\
Tricuspid e' & -0.08 & 0.43 \\
Tricuspid Sm' & 0.08 & 0.38 \\
LV mass Index & 0.30 & 0.003 \\
\hline
\end{tabular}

Multivariate regresion analyses

\begin{tabular}{lrl}
\hline \multicolumn{1}{c}{$r^{2}=0.25$} & $T$ & $p$ \\
\hline Mitral Tei Index & 2.14 & 0.014 \\
Mitral e' & -0.52 & 0.6 \\
LV mass Index & 2.16 & 0.001 \\
LA volume Index & 3.21 & 0.002 \\
\hline
\end{tabular}

LVEF, Left ventricular ejection fraction; LA, Left atrium; LV, Left ventricular; Dec T, Deceleration time. fasting glucose levels $(r=0.27, p=0.008)$, BMI $(r=0.8$, $p<0.001)$, waist circumferences $(r=0.75, p<0.001)$, MTI $(r=0.27, p=0.005)$, or mitral annulus TDI $\mathrm{E}^{\prime}$ velocities $(r=0.19, p=0.04)$. Multivariate regression analysis demonstrated that EFT was associated with BMI $(t=5.28, p=$ $0.001)$, waist circumferences $(t=10.0, p=0.001)$ and TG levels $(t=2.07, p=0.04)$. Considering echocardiographic parameters, multivariate analysis demonstrated the association of EFT with LVMI $(r=2.16, p=0.01)$, LAVI $(r=3.21$, $p=0.002)$, and MTI $(r=2.39, p=0.019)$ (Tables 2 and 3$)$.

\section{Discussion}

In the present study, a significant correlation was noted between EFT and MS in premenopausal woman. Waist circumference, fasting blood glucose, TG and HDL levels as the components of MS showed strong correlation with EFT. On the other hand, left ventricular systolic and diastolic parameters were also correlated well with EFT. Moreover, significant correlation was seen between CRP and EFT. These findings suggest that measuring EFT is invaluable for determining the risk in healthy individuals with MS.

Epicardial fat is a visceral fat tissue, deposited around the heart on the free wall of the right ventricle, left ventricular apex, and atrium. Previous reports indicated that epicardial fat tissue was strongly correlated with abdominal fat deposits (Marchington et al. 1989; Sacks and Fain 2007). The biochemical properties of epicardial fat tissue suggest its possible role as a cardiovascular risk factor. According to biomolecular studies in humans, epicardial fat tissue is metabolically active and an important source of both proinflammatory adipokines, such as tumor necrosis factor- $\alpha$, interleukin 1, interleukin 6 and nerve growth factor, and anti-inflammatory adipokines, such as adiponectin and adrenomedullin (Iacobellis et al. 2005b; Baker et al. 2006; Iacobellis and Barbaro 2008). EFT has also been shown to be related to markers of insulin resistance and inflammation (Karastergiou et al. 2010). Epicardial fat tissue has unique endocrine and paracrine functions that affect the cardiac autonomic system. It has also been shown that EFT was related to blunted heart rate recovery, which predicts autonomic dysfunction in patients with MS (Sengul and Duman 2011).

Iacobellis et al. (2003a) measured epicardial fat by echocardiography for the first time. They revealed an excellent correlation of echocardiographic EFT by echocardiography with abdominal fat and epicardial fat measurements by MRI. Echocardiographically measured epicardial fat could provide a highly reliable measure of true visceral fat content and its greatest utility as a less expensive method for precise quantification of EFT (Iacobellis et al. 2005b).

Obesity seems to be a predisposing factor for the accumulation of excess epicardial fat (Schejbal 1989). We showed strong correlation between EFT and BMI, indicating that obesity is related with increased EFT. Obesity is an important risk factor for atherosclerotic cardiovascular dis- 
ease (Hubert et al. 1983; Manson et al. 1990; Janssen et al. 2002; Ridker et al. 2002). Relationship between MS and coronary heart disease (CHD) has already been proved (Janssen et al. 2002; Ridker et al. 2002; Engeli et al. 2003; Duman 2012). In our study, we determined significant correlation between four important parameters of MS (waist circumference, TG levels, blood fasting glucose, and HDL levels) and EFT. Unlike other studies we determined strong correlation between EFT and CRP, which is an acute phase reactant and whose strong relationship with CHD has been established (Ridker et al. 2002; Engeli et al. 2003). On the other hand, we found that CRP was significantly elevated with an increase in EFT in this healthy female population who were prone to MS, but not diagnosed as coronary heart disease. Epicardial fat tissue is usually located in close proximity to the coronary vasculature. Such distinguishing biochemical properties and the unique anatomic location of epicardial fat tissue were hypothesized to enhance its paracrine role in the development of coronary atherosclerosis (Rosito et al. 2008; Gorter et al. 2008). A local interaction between epicardial fat and the nearby coronary arteries may explain the association between epicardial fat and inflammation, as evident with high CRP levels in our study. This finding suggests that EFT could be an early sign of endothelial dysfunction at subclinical stage and could be a predictor of the inflammation that causes CHD.

The cardiac structural changes in obesity like increased LV mass and LA volume as well as systolic and diastolic dysfunction have already been supported by previous data (Laaban et al. 2002; Wong et al. 2004). EFT could be a marker of cardiac adiposity and obesity; therefore it could play an important role in obesity-related LV abnormalities. EFT was also found correlated with LV mass, LA dimension, and diastolic function. Nevertheless, the close anatomical and functional relationship of epicardial fat tissue with the adjacent myocardium could lead to local and paracrine interactions between these tissues (Iacobellis et al. 2006). MTI is a new echocardiographic parameter that correlates better with invasive measurements and could be used to evaluate both systolic and diastolic functions (Tei et al. 1995). Besides increased LVMI and LAVI measurements, MTI were also calculated significantly increased in this healthy population as a novel finding.

In conclusion, measuring EFT is a new and easily applicable method. Increased epicardial fat tissue is an early finding of inflammation that is metabolically active and could be a predictor of subclinical cardiac deterioration in MS. These subclinical cardiac changes could be determined by increased MTI, LVMI and LAVI. CRP as an index of CHD is in a close relationship with EFT. Considering these close relationships, EFT could be interpreted as an early parameter of MS and premature atherosclerosis.

\section{Conflict of Interest}

The authors don't report any conflict of interest regarding this work.

\section{References}

Ahn, S.G., Lim, H.S., Joe, D.Y., Kang, S.J., Choi, B.J., Choi, S.Y., Yoon, M.H., Hwang, G.S., Tahk, S.J. \& Shin, J.H. (2008) Relationship of epicardial adipose tissue by echocardiography to coronary artery disease. Heart, $\mathbf{9 4 ,}$ e7.

Baker, A.R., Silva, N.F., Quinn, D.W., Harte, A.L., Pagano, D., Bonser, R.S., Kumar, S. \& McTernan, P.G. (2006) Human epicardial adipose tissue expresses a pathogenic profile of adipocytokines in patients with cardiovascular disease. Cardiovasc. Diabetol., 5, 1-7.

Devereux, R.B. \& Reichek, N. (1977) Echocardiographic determination of left ventricular mass in man. Anatomic validation of the method. Circulation, 55, 613-618.

Ding, J., Hsu, F.C., Harris, T.B., Liu, Y., Kritchevsky, S.B., Szklo, M., Ouyang, P., Espeland, M.A., Lohman, K.K., Criqui, M.H., Allison, M., Bluemke, D.A. \& Carr, J.J. (2009) The association of pericardial fat with incident coronary heart disease: the Multi-Ethnic Study of Atherosclerosis (MESA). Am. J. Clin. Nutr., 90, 499-504.

Duman, D. (2012) Is increased epicardial fat thickness a marker of the presence of severe coronary artery disease? Anadolu Kardiyol. Derg., 12, 206-207.

Engeli, S., Feldpausch, M., Gorzelniak, K., Hartwig, F., Heintze, U., Janke, J., Mohlig, M., Pfeiffer, A.F., Luft, F.C. \& Sharma, A.M. (2003) Association between adiponectin and mediators of inflammation in obese women. Diabetes, 52, 942-947.

Gottdiener, J.S., Bednarz, J., Devereux, R., Gardin, J., Klein, A., Manning, W.J., Morehead, A., Kitzman, D., Oh, J., Quinones, M., Schiller, N.B., Stein, J.H. \& Weissman, N.J. (2004) American Society of Echocardiography recommendations for use of echocardiography in clinical trials. J. Am. Soc. Echocardiogr., 17, 1086-1119.

Gorter, P.M., De Vos, A.M., Van Der Graaf, Y., Stella, P.R., Doevendans, P.A., Meijs, M.F., Prokop, M. \& Visseren, F.L. (2008) Relation of epicardial and pericoronary fat to coronary atherosclerosis and coronary artery calcium in patients undergoing coronary angiography. Am. J. Cardiol., 102, 380-385.

Hubert, H.B., Feinleib, M., McNamara, P.M. \& Castelli, W.P. (1983) Obesity as an independent risk factor for cardiovascular disease: a 26-year followup of participants in the Framingham Heart Study. Circulation, 67, 968-977.

Iacobellis, G., Assael, F., Ribaudo, M.C., Zappaterreno, A., Alessi, G., Di Mario, U. \& Leonetti, F. (2003a) Epicardial fat from echocardiography: a new method for visceral adipose tissue prediction. Obes. Res., 11, 304-310.

Iacobellis, G. \& Barbaro, G. (2008) The double role of epicardial adipose tissue as pro- and anti-inflammatory organ. Horm. Metab. Res., 40, 442-445.

Iacobellis, G., Corradi, D. \& Sharma, A.M. (2005a) Epicardial adipose tissue: anatomic, biomolecular and clinical relationships with the heart. Nat. Clin. Pract. Cardiovasc. Med., 2, 536-543.

Iacobellis, G., Pistilli, D., Gucciardo, M., Leonetti, F., Miraldi, F., Brancaccio, G., Gallo, P. \& Di Gioia, C.R. (2005b) Adiponectin expression in human epicardial adipose tissue in vivo is lower in patients with coronary artery disease. Cytokine, 29, 251-255.

Iacobellis, G., Pond, C.M. \& Sharma, A.M. (2006) Different "weight" of cardiac and general adiposity in predicting left ventricle morphology. Obesity (Silver Spring), 14, 1679-1684.

Iacobellis, G., Ribaudo, M.C., Assael, F., Vecci, E., Tiberti, C., Zappaterreno, A., Di Mario, U. \& Leonetti, F. (2003b) Echo- 
cardiographic epicardial adipose tissue is related to anthropometric and clinical parameters of metabolic syndrome: a new indicator of cardiovascular risk. J. Clin. Endocrinol. Metab., 88, 5163-5168.

Janssen, I., Katzmarzyk, P.T. \& Ross, R. (2002) Body mass index, waist circumference, and health risk: Evidence in support of current National Institutes of Health guidelines. Arch. Intern. Med., 162, 2074-2079.

Karastergiou, K. \& Mohamed-Ali, V. (2010) The autocrine and paracrine roles of adipokines. Mol. Cell Endocrinol., 318, 69-78.

Laaban, J.P., Pascal-Sebaoun, S., Bloch, E., Orvoën-Frija, E., Oppert, J.M. \& Huchon, G. (2002) Left ventricular systolic dysfunction in patients with obstructive sleep apnea syndrome. Chest, 122, 1133-1138.

Lang, R.M., Bierig, M., Devereux, R.B., Flachskampf, F.A., Foster, E., Pellikka, P.A., Picard, M.H., Roman, M.J., Seward, J., Shanewise, J.S., Solomon, S.D., Spencer, K.T., Sutton, M.S. \& Stewart, W.J. (2005) Recommendations for chamber quantification: a report from the American Society of Echocardiography's Guidelines and Standards Committee and the Chamber Quantification Writing Group, developed in conjunction with the European Association of Echocardiography, a branch of the European Society of Cardiology. J. Am. Soc. Echocardiogr., 18, 1440-1463.

Manson, J.E., Colditz, G.A., Stampfer, M.J., Willett, W.C., Rosner, B., Monson, R.R., Speizer, F. \& Hennekes, C.H. (1990) A prospective study of obesity and risk of coronary heart disease in women. N. Engl. J. Med., 322, 882-889.

Marchington, J.M., Mattacks, C.A. \& Pond, C.M. (1989) Adipose tissue in the mammalian heart and pericardium: structure, foetal development and biochemical properties. Comp. Biochem. Physiol. B. Comp. Biochem., 94, 225-232.

National Cholesterol Education Program (NCEP) Expert Panel on Detection, Evaluation, and Treatment of High Blood Cholesterol in Adults (Adult Treatment Panel III) (2002) Third Report of the National Cholesterol Education Program (NCEP) Expert Panel on Detection, Evaluation, and Treatment of High Blood Cholesterol in Adults (Adult Treatment Panel III) final report. Circulation, 106, 3143-3421.

Pritchett, A.M., Jacobsen, S.J., Mahoney, D.W., Rodeheffer, R.J., Bailey, K.R. \& Redfield, M.M. (2003) Left atrial volume as an index of left atrial size: a population-based study. J. Am. Coll. Cardiol., 41, 1036-1043.

Ridker, P.M., Rifai, N., Rose, L., Buring, J.E. \& Cook, N.R. (2002) Comparison of C-reactive protein and low-density lipoprotein cholesterol levels in the prediction of first cardiovascular events. N. Engl. J. Med., 347, 1557-1565.

Rosito, G.A., Massaro, J.M., Hoffmann, U., Ruberg, F.L., Mahabadi, A.A., Vasan, R.S., O'Donnell, C.J. \& Fox, C.S. (2008) Pericardial fat, visceral abdominal fat, cardiovascular disease risk factors, and vascular calcification in a communitybased sample: the Framingham Heart Study. Circulation, 117, 605-613.

Sacks, H.S. \& Fain, J.N. (2007) Human epicardial adipose tissue: a review. Am. Heart J., 153, 907-917.

Schejbal, V. (1989) Epicardial fatty tissue of the right ventricle morphology, morphometry and functional significance. Pneumologie, 43, 490-499.

Sengul, C. \& Duman, D. (2011) The association of epicardial fat thickness with blunted heart rate recovery in patients with metabolic syndrome. Tohoku J. Exp. Med., 224, 257-262.

Taguchi, R., Takasu, J., Itani, Y., Yamamoto, R., Yokoyama, K., Watanabe, S. \& Masuda, Y. (2001) Pericardial fat accumulation in men as a risk factor for coronary artery disease. Atherosclerosis, 157, 203-209.

Tei, C., Ling, L.H., Hodge, D.O., Bailey, K.R., Oh, J.K., Rodeheffer, R.J., Tajik, A.J. \& Seward, J.B. (1995) New index of combined systolic and diastolic myocardial performance: a simple and reproducible measure of cardiac function-a study in normals and dilated cardiomyopathy. J. Cardiol., 26, 357-366.

Wong, C.Y., O’Moore-Sullivan, T., Leano, R., Byrne, N., Beller, E. \& Marwick, T.H. (2004) Alterations of left ventricular myocardial characteristics associated with obesity. Circulation, 110, 3081-3087. 